\title{
The Warsaw Proposal for the Use of Extended Selection Criteria in Liver Transplantation for Hepatocellular Cancer
}

\author{
Michał Grąt, MD, PhD ${ }^{1}$, Karolina M. Wronka, MD ${ }^{1}$, Jan Stypułkowski ${ }^{1}$, Emil Bik ${ }^{1}$, Maciej Krasnodębski, MD ${ }^{1}$, \\ Lukasz Masior, $\mathrm{MD}^{1}$, Zbigniew Lewandowski, $\mathrm{PhD}^{2}$, Karolina Grat, $\mathrm{MD}^{3}$, Waldemar Patkowski, MD, $\mathrm{PhD}^{1}$, and \\ Marek Krawczyk, MD, PhD ${ }^{1}$ \\ ${ }^{1}$ Department of General, Transplant and Liver Surgery, Medical University of Warsaw, Warsaw, Poland; ${ }^{2}$ Department of \\ Epidemiology, Medical University of Warsaw, Warsaw, Poland; ${ }^{3}$ Second Department of Clinical Radiology, Medical \\ University of Warsaw, Warsaw, Poland
}

\begin{abstract}
Background. Combination of the University of California, San Francisco (UCSF) and the up-to-7 criteria with alpha-fetoprotein (AFP) cutoff of $100 \mathrm{ng} / \mathrm{ml}$ was proposed as the Warsaw expansion of the Milan criteria in selection of hepatocellular cancer (HCC) patients for liver transplantation. The purpose of this retrospective study was to validate this proposal.
\end{abstract}

Methods. A total of $240 \mathrm{HCC}$ patients after liver transplantation were included. Recurrence-free survival and overall survival at 5 years were set as the primary and secondary outcome measures, respectively.

Results. The Warsaw expansion increased transplant eligibility rate by $20.3 \%$. AFP $>100 \mathrm{ng} / \mathrm{ml}$ significantly increased the recurrence risk in patients within the Milan criteria $(p=0.025)$ and in those beyond, yet within either the UCSF or the up-to-7 criteria $(p<0.001)$. Recurrencefree survival at 5 years was $90.8 \%$ for patients within the Milan criteria, $100.0 \%$ in patients within the Warsaw expansion, $54.9 \%$ in patients beyond the Warsaw expansion but within either the UCSF or the up-to-7 criteria, and $45.1 \%$ in patients beyond both the UCSF and the up-to-7 criteria $(p<0.001)$. The corresponding overall survival rates were $71.6,82.4,64.3$, and $55.3 \%$, respectively ( $p=0.027$ ).

Conclusions. The Warsaw expansion of the Milan criteria substantially increases the recipient pool without compromising outcomes.

(C) The Author(s) 2016. This article is published with open access at Springerlink.com

First Received: 19 May 2016;

Published Online: 16 August 2016

M. Grạt, MD, PhD

e-mail: michal.grat@gmail.com
Liver transplantation is the optimal treatment for selected patients with hepatocellular cancer (HCC) and liver cirrhosis. ${ }^{1,2}$ Despite available evidence for the superiority of long-term outcomes of patients undergoing liver transplantation as a first-line management over those undergoing liver resection or salvage liver transplantation, its use remains limited by the worldwide shortage of donors. $^{3-8}$ Accordingly, the restrictive Milan criteria (1 tumor $<5 \mathrm{~cm}$ or $2-3$ tumors $<3 \mathrm{~cm}$ each combined with the absence of extrahepatic lesions or macrovascular invasion) introduced two decades ago are still the gold standard in selecting patients eligible for transplantation, despite the increasing number of reports on the noninferiority of their modest expansion. ${ }^{9-13}$ Of numerous morphology-based expansion proposals, the University of California, San Francisco criteria (1 tumor $<6.5 \mathrm{~cm}$ or $2-3$ tumors $<4.5 \mathrm{~cm}$ with total tumor diameter $<8 \mathrm{~cm}$ ) and the up-to-7 criteria (sum of number of tumors and size of the largest tumor in cm not exceeding 7) appear most popular and widely evaluated. ${ }^{14,15}$ However, the risk of posttransplant recurrence is dependent upon morphological tumor burden and long-term survival of patients undergoing liver transplantation for $\mathrm{HCC}$ is inferior to those with benign indications even with the widespread use of Milan criteria. ${ }^{16-18}$ Therefore, expansion of limits for tumor size and number is subject to criticism. ${ }^{19-21}$

Considering the increasing evidence for the independent role of serum tumor markers, particularly alpha-fetoprotein (AFP), in prediction of posttransplant tumor recurrence, modification of the morphology-based criteria using these markers of biological aggressiveness seems not only justified, but also necessary. ${ }^{22-25}$ Notably, while serum tumor markers already have been included in several criteria proposals utilized in Asian countries, utilization of such 
strategy is uncommon in European and American countries, besides a recent French AFP model by Duvoux et al. and the AFP total tumor volume (TTV) criteria by Toso et al. $^{26-32}$ In view of geographical discrepancies in the selection criteria and following a detailed set of analyses of the associations between pretransplant AFP and the risk of posttransplant tumor recurrence, AFP was included in the Warsaw proposal for safe extension of Milan criteria. Briefly, it defines patient eligibility either (1) by fulfillment of the Milan criteria, irrespective of pretransplant AFP, or (2) fulfillment of the up-to-7 or UCSF criteria and AFP $<100 \mathrm{ng} / \mathrm{ml} .{ }^{33}$ In the initial study, the latter subgroup was characterized by the ideal 5-year, recurrence-free survival of $100 \%$. This proposal was started to be taken under consideration during selection of HCC patients for liver transplantation since the beginning of 2013. Therefore, the purpose of the present study was to evaluate the efficacy of the Warsaw proposal for selection of HCC patients for liver transplantation in provision of acceptable low-risk of posttransplant recurrence.

\section{METHODS}

A total of 1602 liver transplantations were performed in the Department of General, Transplant and Liver Surgery at the Medical University of Warsaw (Poland) during the period between December 1989 and April 2015. This retrospective cohort study was performed using the data of 240 patients after liver transplantation for HCC between January 2001 and April 2015. The study protocol was approved by the local ethics committee of the Medical University of Warsaw.

Tumor recurrence at 5-year, follow-up period was set as a primary endpoint of the study, with 5-year recurrence free survival (RFS) being the primary outcome measure. The 5-year RFS was calculated as the time from liver transplantation until diagnosis of recurrence and was censored at the date of last follow-up or death from a nonmalignant cause. The secondary endpoint was patient death at 5 years (irrespective of the cause), which was used to calculate overall survival (OS, secondary outcome measure). Details on the center's experience, perioperative management, operative technique, immunosuppression protocol, and long-term follow-up were provided elsewhere. ${ }^{34,35}$

Primary and secondary outcome measures were evaluated separately in patients (1) within the Milan criteria, (2) beyond the Milan criteria but within the up-to-7 or the UCSF criteria with AFP $<100 \mathrm{ng} / \mathrm{ml}$ (Warsaw proposal for extension of the Milan criteria), (3) $>100 \mathrm{ng} / \mathrm{ml}$, and (4) in patients beyond both the UCSF and the up-to-7 criteria. The particular selection criteria and last pretransplant AFP, as primary variables of interest, were assessed as risk factors for tumor recurrence at 5 years. This was done both in a series of univariable analyses and in multivariable analyses, following adjustment for the impact of other risk factors for rumor recurrence.

Quantitative variables were presented as medians (interquartile ranges) and qualitative variables were presented as numbers (percentages). Kruskal-Wallis test and Chisquare test were used for intergroup comparisons, as appropriate. Kaplan-Meier estimator was used to calculate survival curves and log-rank test was used for the corresponding comparisons. Reverse Kaplan-Meier method was used to calculate median follow-up. Cox proportional hazards regression models were used to evaluate the risk factors for tumor recurrence. Due to the number of events, a series of three variable models were used instead of a one multivariable model. Hazard ratios (HRs) were presented with $95 \%$ confidence intervals $(95 \% \mathrm{CI})$. The level of statistical significance was set to 0.05 . STATISTICA version 12 software (StatSoft Inc., Tulsa, OK) was applied for computing statistical analyses.

\section{RESULTS}

Of 240 patients included in the study, $143(59.6 \%)$ were within the Milan criteria, $171(71.3 \%)$ were within the UCSF criteria, and 181 (75.4\%) were within the up-to-7 criteria. Compared with the fulfillment of Milan criteria, utilization of Warsaw extension results in $20.3 \%$ increase in the number of potential HCC recipients (143-172). Comparisons of baseline characteristics between patients within the Milan criteria, beyond the Milan criteria but within the UCSF or the up-to-7 criteria with AFP $<100 \mathrm{ng} / \mathrm{ml}$ (Warsaw extension), and beyond that limits are presented in Table 1 . Besides fulfillment of the particular selection criteria, significant differences between these subgroups were found with respect to AFP concentration $(p<0.001)$, number of tumors $(p<0.001)$, size of the largest tumor $(p<0.001)$, total tumor volume $(p<0.001)$, microvascular invasion $(p<0.001)$, tumor differentiation $(p=0.037)$, and neoadjuvant treatment $(p=0.040)$. Median follow-up was 34 months. In the entire study cohort, 31 patients developed tumor recurrence within 5 years posttransplantation with the RFS rates of $92.3 \%$ at 1 year, $84.8 \%$ at 3 years, and $79.5 \%$ at 5 years. There were 49 deaths in the 5 -year posttransplant period with the corresponding OS rates of 89.0, 79.8, and $68.8 \%$, respectively.

According to the results of univariable analyses, the Milan criteria $(p<0.001)$, the UCSF criteria $(p<0.001)$, the up-to-7 criteria $(p<0.001)$, and the last pretransplant AFP $(p<0.001)$ were significantly associated with the risk of posttransplant tumor recurrence (Table 2). Other risk factors comprised younger recipient age $(p=0.034)$, 
TABLE 1 Comparison of baseline characteristics between patients within the Milan criteria, patients beyond Milan criteria but within the UCSF or up-to-7 criteria with AFP $<100 \mathrm{ng} / \mathrm{ml}$ (the Warsaw extension), and patients beyond the Warsaw extension

\begin{tabular}{|c|c|c|c|c|}
\hline Characteristics & $\begin{array}{l}\text { Within Milan criteria } \\
(n=143)\end{array}$ & $\begin{array}{l}\text { Warsaw extension } \\
(n=29)\end{array}$ & $\begin{array}{l}\text { Beyond Warsaw extension } \\
(n=68)\end{array}$ & $P$ \\
\hline Recipient gender & & & & 0.251 \\
\hline Male & $100(69.9 \%)$ & $19(65.5 \%)$ & $54(79.4 \%)$ & \\
\hline Female & $43(30.1 \%)$ & $10(34.5 \%)$ & $14(20.6 \%)$ & \\
\hline Recipient age (yr) & $57(52-61)$ & $58(53-61)$ & $56(52-61)$ & 0.573 \\
\hline MELD & $11(9-13)$ & $9(7-12)$ & $11(8-14)$ & 0.183 \\
\hline $\mathrm{HCV}$ infection & $100(69.9 \%)$ & $20(69.0 \%)$ & $49(72.1 \%)$ & 0.935 \\
\hline HBV infection & $64(44.8 \%)$ & $12(41.4 \%)$ & $26(38.2 \%)$ & 0.664 \\
\hline Within UCSF criteria & $143(100.0 \%)$ & $19(65.5 \%)$ & $9(13.2 \%)$ & $<0.001$ \\
\hline Within up-to-7 criteria & $143(100.0 \%)$ & $27(93.1 \%)$ & $11(16.2 \%)$ & $<0.001$ \\
\hline Number of tumors & $1(1-2)$ & $2(1-3)$ & $4(2-7)$ & $<0.001$ \\
\hline Size of the largest tumor (mm) & $25(15-33)$ & $40(33-51)$ & $45(35-60)$ & $<0.001$ \\
\hline Total tumor volume $\left(\mathrm{cm}^{3}\right)$ & $8(2-22)$ & $44(28-80)$ & $88(49-131)$ & $<0.001$ \\
\hline Pretransplant AFP (ng/ml) & $13(5-58)$ & $11(6-21)$ & $114(14-914)$ & $\begin{array}{l}<0.001 \\
<0.001\end{array}$ \\
\hline \multicolumn{5}{|l|}{ Pretransplant AFP } \\
\hline$>100 \mathrm{ng} / \mathrm{ml}$ & $28(20.4 \%)$ & $0(0.0 \%)$ & $34(50.7 \%)$ & \\
\hline$<100 \mathrm{ng} / \mathrm{ml}$ & $109(79.6 \%)$ & $29(100.0 \%)$ & $33(49.3 \%)$ & \\
\hline Poor tumor differentiation & $9(6.3 \%)$ & $3(10.3 \%)$ & $12(17.6 \%)$ & 0.037 \\
\hline Microvascular invasion & $22(15.5 \%)$ & $11(39.3 \%)$ & $34(52.3 \%)$ & $<0.001$ \\
\hline Neoadjuvant treatment & $57(39.9 \%)$ & $19(65.5 \%)$ & $30(44.1 \%)$ & 0.040 \\
\hline Total ischemic time (hr) & $9.0(8.0-10.4)$ & $9.8(8.5-10.5)$ & $9.3(8.0-10.3)$ & 0.553 \\
\hline $\begin{array}{l}\text { Intraoperative PRBC transfusions } \\
\text { (units) }\end{array}$ & $3(0-6)$ & $3(2-5)$ & $4(2-6)$ & 0.420 \\
\hline Intraoperative FFP transfusions (units) & $6(4-10)$ & $7(5-10)$ & $7(5-10)$ & 0.232 \\
\hline
\end{tabular}

Data are presented as medians (interquartile ranges) or numbers (percentages)

UCSF University of California, San Francisco; $A F P$ alpha-fetoprotein; MELD model for end-stage liver disease; $H C V$ hepatitis $\mathrm{C}$ virus; $H B V$ hepatitis B virus; $P R B C$ packed red blood cells; FFP fresh frozen plasma

presence of microvascular invasion $(p=0.029)$, poor tumor differentiation $(p=0.013)$, and morphological tumor features: number of tumors $(p<0.001)$, size of the largest tumor $(p=0.001)$, and total tumor volume $(p<0.001)$. Notably, AFP $>100 \mathrm{ng} / \mathrm{ml}$ was a significant risk factor for tumor recurrence in patients within the Milan criteria (HR $7.00 ; 95 \%$ CI $1.28-38.3 ; p=0.025)$ and in those beyond the Milan criteria but within either the UCSF or the up-to-7 criteria (100.0 vs. $54.9 \%, p<0.001)$, however not in patients beyond both the UCSF and the up-to-7 criteria (HR 1.73; $95 \%$ CI 0.70-4.29; $p=0.236$ ). Furthermore, a series of multivariable (3-factor) analyses revealed that nonfulfillment of each of the analyzed criteria and AFP $>100 \mathrm{ng} / \mathrm{ml}$ were independent risk factors for worse 5-year RFS (Table 3). As each of the three particular selection criteria are solely defined by morphological tumor features, number of tumors, size of the largest tumor and total tumor volume were not included in the multivariable models.
Patients within the Milan criteria exhibited a 5-year RFS rate of $90.8 \%$, significantly higher than that observed for patients beyond the Milan criteria $(64.2 \%, p<0.001$; Fig. 1a). The OS rates in the corresponding subgroups were $71.6 \%$ and $65.4 \%$, respectively $(p=0.055$; Fig. $1 b)$. Compared with patients within the Milan criteria, patients beyond Milan but within either the UCSF or the up-to-7 criteria exhibited nonsignificantly lower 5-year RFS of $88.0 \%(p=0.350)$, yet higher than that observed for patients beyond the UCSF and the up-to-7 criteria (45.1\%, $p=0.004$; Fig. 1c). The only significant difference between these subgroups with respect to OS was found between patients within the Milan criteria and those beyond the up-to-7 and the UCSF criteria (71.6 vs. $55.3 \%$, $p=0.012$; Fig. 1d). Notably, RFS at 5 years for patients beyond the Milan criteria but within their Warsaw extension was $100 \%$ compared with $90.8 \%$ in patients within the Milan criteria $(p=0.161), 54.9 \%$ in patients beyond 
TABLE 2 Results of univariable analyses of risk factors for 5-year tumor recurrence

\begin{tabular}{lllr}
\hline Factors & Hazard ratio & $95 \%$ confidence interval & $P$ \\
Male recipient gender & 0.84 & $0.39-1.83$ & 0.663 \\
Recipient age & 0.97 & $0.93-0.99$ & 0.034 \\
MELD & 0.99 & $0.92-1.07$ & 0.814 \\
HCV infection & 1.00 & $0.47-2.13$ & 0.998 \\
HBV infection & 1.41 & $0.69-2.85$ & 0.343 \\
Within Milan criteria & 0.22 & $0.10-0.48$ & $<0.001$ \\
Within UCSF criteria & 0.25 & $0.12-0.50$ & $<0.001$ \\
Within up-to-7 criteria & 0.17 & $0.08-0.36$ & $<0.001$ \\
Number of tumors & 1.31 & $1.18-1.44$ & $<0.001$ \\
Size of the largest tumor & 1.02 & $1.01-1.04$ & 0.001 \\
Total tumor volume & 1.01 & $1.01-1.02$ & $<0.001$ \\
Pre-transplant AFP $>100 \mathrm{ng} / \mathrm{ml}$ & 4.30 & $2.05-9.00$ & $<0.001$ \\
Poor tumor differentiation & 2.90 & $1.25-6.73$ & 0.013 \\
Microvascular invasion & 2.23 & $1.09-4.57$ & 0.029 \\
Neoadjuvant treatment & 1.36 & $0.67-2.76$ & 0.393 \\
Total ischemic time & 1.15 & $0.94-1.39$ & $0.93-1.07$ \\
Intraoperative PRBC transfusions & 1.00 & $0.90-1.06$ & 0.172 \\
Intraoperative FFP transfusions & 0.98 & 0.986 \\
\hline Hazard & & 0.626 \\
\end{tabular}

Hazard ratios were given per: 1 year increase for recipient age; 1 point increase for model for end-stage liver disease; 1 tumor more for number of tumors; $1 \mathrm{~mm}$ increase for the size of the largest tumor; $10 \mathrm{~cm}^{3}$ increase for total tumor volume; $1 \log _{\mathrm{e}}$ increase for alpha-fetoprotein; $1 \mathrm{~h}$ increase for total ischemic time; and 1 unit increase for packed red blood cells and fresh frozen plasma transfusions

$M E L D$ model for end-stage liver disease; $H C V$ hepatitis $\mathrm{C}$ virus; $H B V$ hepatitis B virus; UCSF University of California, San Francisco; $A F P$ alpha-fetoprotein; $P R B C$ packed red blood cells; FFP fresh frozen plasma

TABLE 3 Multivariable (3-factor) analyses of the associations between fulfillment of particular selection criteria and AFP over $100 \mathrm{ng} / \mathrm{ml}$ and the risk of posttransplant tumor recurrence

\begin{tabular}{|c|c|c|c|c|c|c|}
\hline \multirow[t]{3}{*}{ Factors } & \multicolumn{6}{|c|}{ The effects of selection criteria fulfillment and AFP on recurrence-free survival adjusted for } \\
\hline & \multicolumn{2}{|c|}{ Microvascular invasion } & \multicolumn{2}{|c|}{ Poor tumor differentiation } & \multicolumn{2}{|l|}{ Recipient age } \\
\hline & $\mathrm{HR}(95 \% \mathrm{CI})$ & $P$ & $\mathrm{HR}(95 \% \mathrm{CI})$ & $P$ & $\mathrm{HR}(95 \% \mathrm{CI})$ & $P$ \\
\hline \multicolumn{7}{|l|}{ Milan criteria and AFP } \\
\hline Within Milan criteria & $0.20(0.08-0.50)$ & $<0.001$ & $0.19(0.08-0.48)$ & $<0.001$ & $0.18(0.07-0.44)$ & $<0.001$ \\
\hline AFP $>100 \mathrm{ng} / \mathrm{ml}$ & $3.98(1.87-8.47)$ & $<0.001$ & $3.56(1.69-7.52)$ & $<0.001$ & $3.46(1.62-7.41)$ & 0.001 \\
\hline \multicolumn{7}{|l|}{ UCSF criteria and AFP } \\
\hline Within UCSF criteria & $0.26(0.12-0.57)$ & $<0.001$ & $0.25(0.11-0.54)$ & $<0.001$ & $0.23(0.10-0.50)$ & $<.001$ \\
\hline AFP >100 ng/ml & $3.83(1.81-8.12)$ & $<0.001$ & $3.31(1.55-7.08)$ & 0.002 & $3.38(1.58-7.22)$ & .002 \\
\hline \multicolumn{7}{|l|}{ Up-to-7 criteria and AFP } \\
\hline Within up-to-7 criteria & $0.19(0.08-0.42)$ & $<0.001$ & $0.17(0.08-0.37)$ & $<0.001$ & $0.18(0.08-0.38)$ & $<.001$ \\
\hline AFP > $100 \mathrm{ng} / \mathrm{ml}$ & $3.60(1.69-7.64)$ & $<0.001$ & $3.18(1.50-6.75)$ & 0.003 & $3.27(1.53-6.99)$ & .002 \\
\hline
\end{tabular}

AFP alpha-fetoprotein; HR hazard ratio; $95 \%$ CI $95 \%$ confidence interval; UCSF University of California, San Francisco

the Milan criteria but within either the UCSF or the up-to-7 criteria and AFP $>100 \mathrm{ng} / \mathrm{ml}(p<0.001)$, and $45.1 \%$ in patients beyond the UCSF and the up-to-7 criteria $(p<0.001 ;$ Fig. 2a). At 5 years, OS was significantly better for both patients within the Milan criteria $(71.6 \%)$ and those beyond the Milan criteria but within their Warsaw extension (82.4\%) compared with patients beyond the UCSF and the up-to-7 criteria $(55.3 \%, p=0.012,0.045$, 

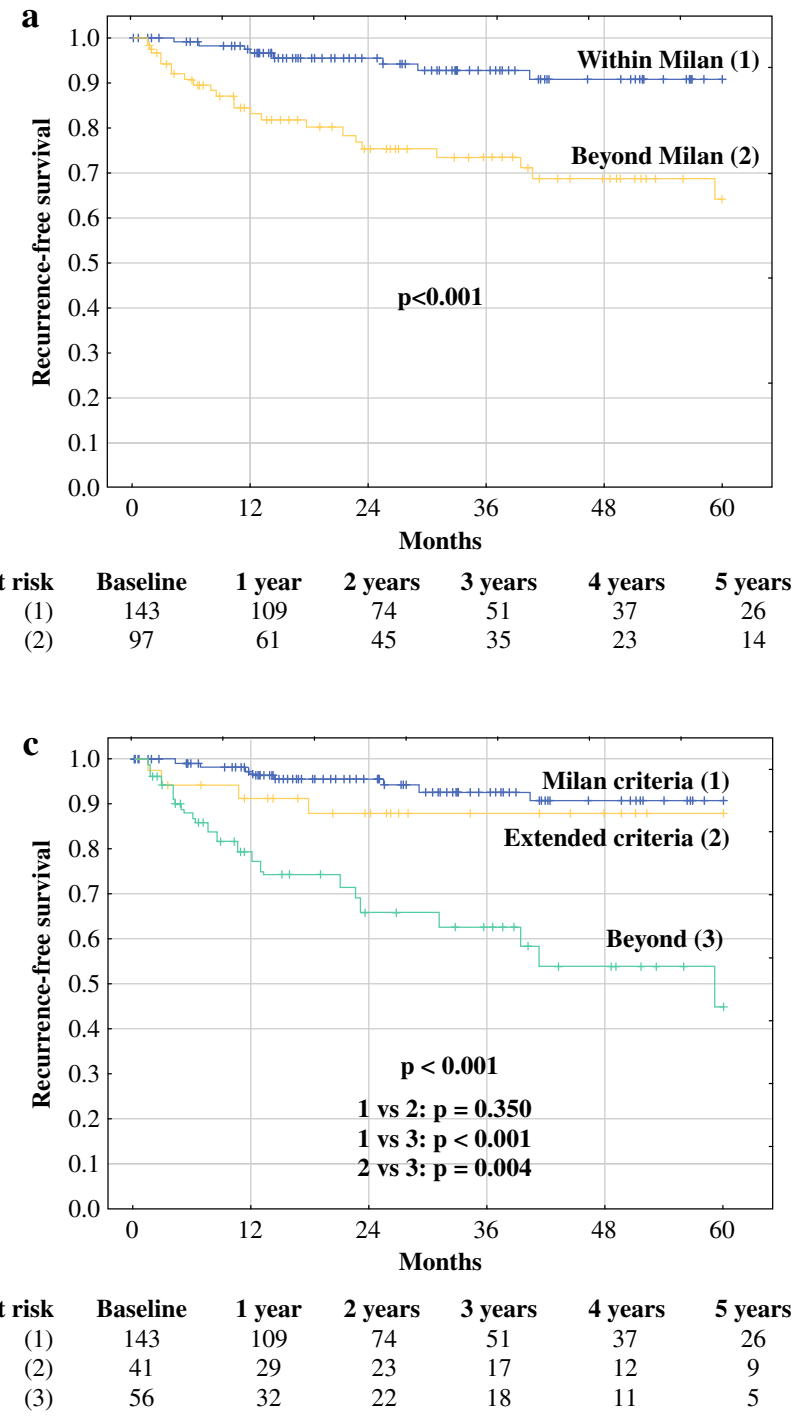

FIG. 1 Recurrence-free survival and overall survival curves in patients within (solid lines) and beyond (dashed lines) the Milan criteria (a, b) and in patients within the Milan criteria (solid lines), beyond the Milan but within either the UCSF or the up-to-7 criteria

respectively; Fig. 2b). Finally, patients within the Warsaw extended criteria exhibited 5-year RFS and OS rates of 92.5 and $73.5 \%$, respectively, compared with the corresponding rates of $47.9 \%(p<0.001)$ and $57.4 \%(p=0.004)$, respectively, in patients beyond these criteria (Fig. 2c, d).

\section{DISCUSSION}

To keep the rates of tumor recurrence after liver transplantation for HCC within the acceptable limits and longterm posttransplant survival of HCC patients comparable to recipients with nonmalignant diseases, the Milan criteria remained the benchmark for assessing patient eligibility for transplantation for the past two decades. The results of
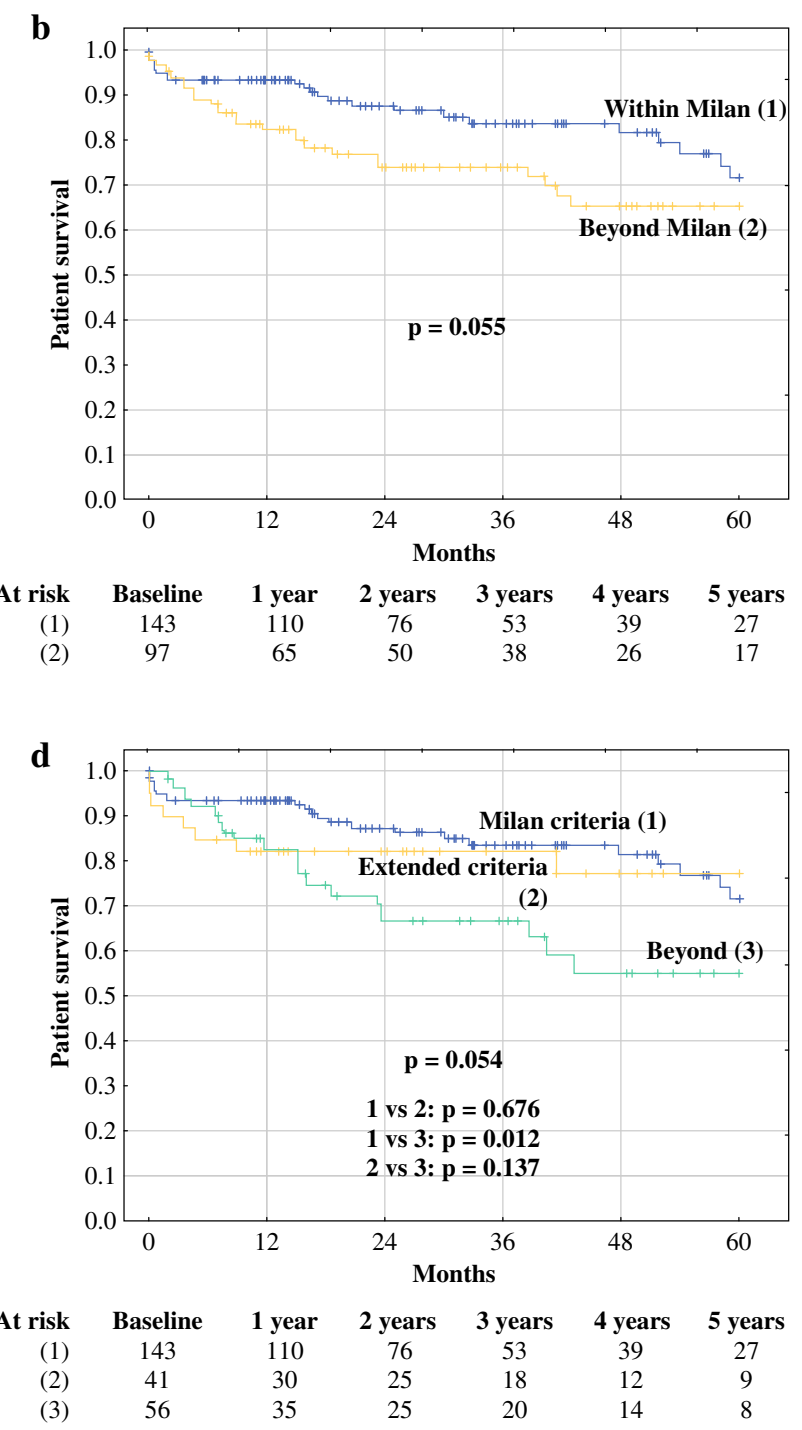

(dashed lines), and beyond (dotted lines) both the UCSF and the upto-7 criteria (c, d). Numbers of patients at risk are presented below the particular graphs

previous study from the authors' department provided the rationale for expansion of these criteria based on the combination of two well-known morphological expansions, namely the UCSF and the up-to-7 criteria, and the biological criterion of AFP $<100 \mathrm{ng} / \mathrm{ml} .^{33}$ The results of the present study provide further evidence on the lack of any negative effects associated with expansion of the Milan criteria into the Warsaw expanded criteria.

The major disadvantage of the proposals for expansion of Milan criteria based only on morphological features is the potential risk of concomitant increase in the risk of posttransplant tumor recurrence. Despite the optimistic results of prospective validation of the UCSF criteria and the results of several retrospective studies indicating 
a

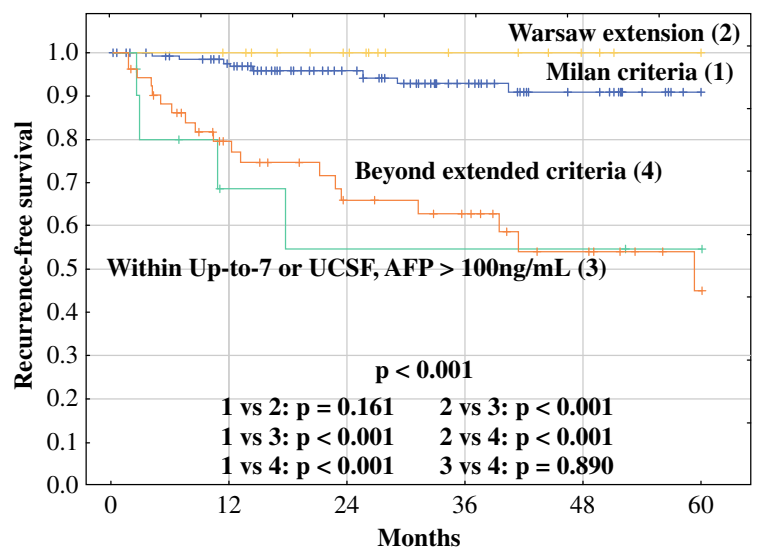

c

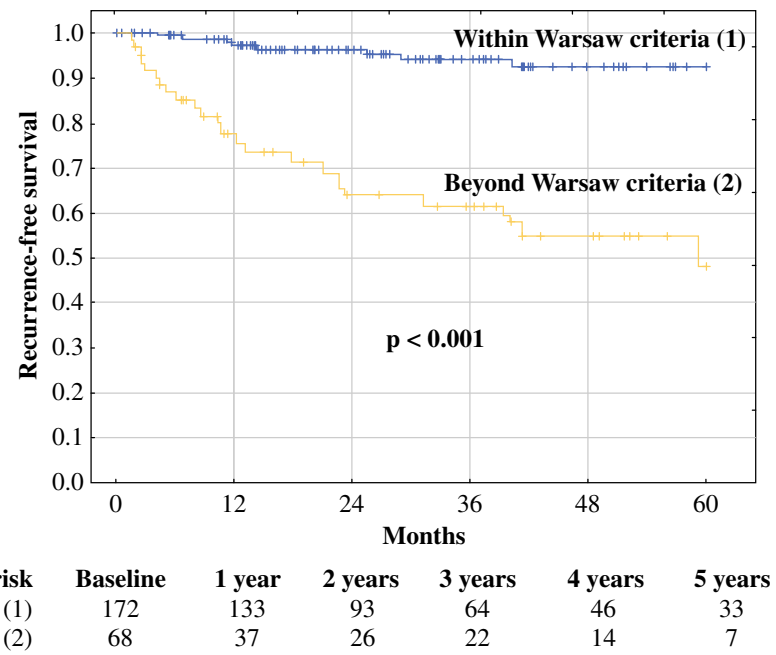

FIG. 2 Recurrence-free survival and overall survival curves in patients within the Milan criteria (solid lines), beyond the Milan criteria but within the Warsaw expansion (short-dashed lines), beyond the Milan criteria and Warsaw expansion but within either the UCSF or the up-to-7 criteria (dotted lines), and beyond both the

acceptable posttransplant outcomes in patients within either of the two expanded criteria, increase in tumor number and size is a well-known factor associated with the recurrence risk. ${ }^{15,36-40}$ In fact, the significant effects of tumor number and size have been the basis for creation of the Metroticket model. ${ }^{15}$ Accordingly, patients beyond Milan but within the limits of the UCSF criteria were previously reported to exhibit inferior long-term outcomes. $^{41,42}$ In the present study, all morphological tumor characteristics were found to be significantly associated with the risk of posttransplant recurrence. Although the

b

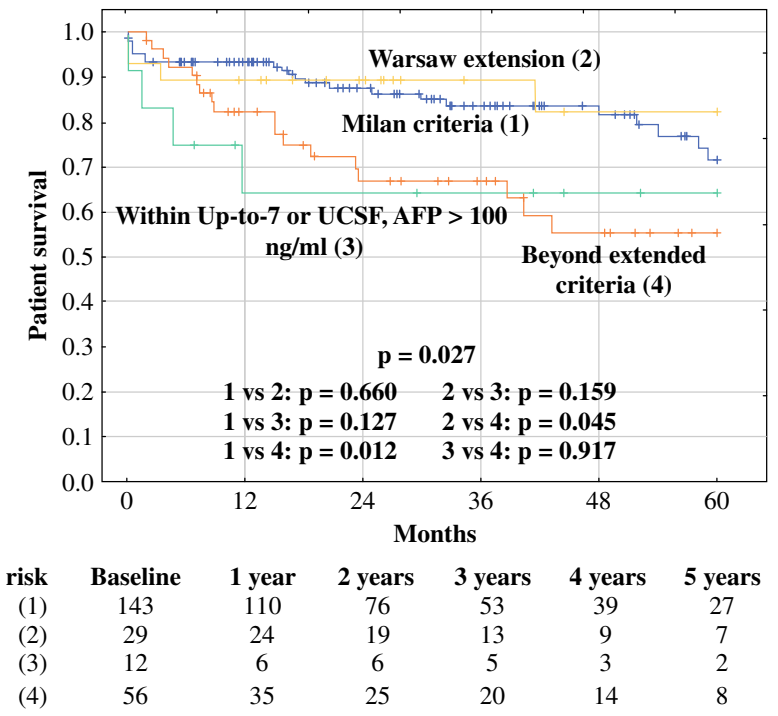

d

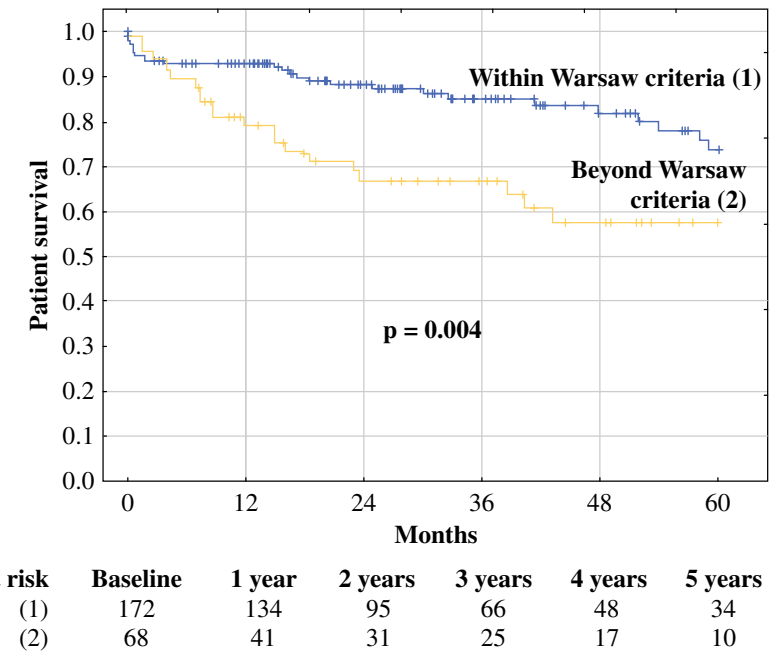

UCSF and the up-to-7 criteria (long-dashed lines) (a, b) and in patients within (solid lines) and beyond (dashed lines) the Warsaw criteria (c, d). Numbers of patients at risk are presented below the particular graphs

long-term outcomes were not significantly compromised in patients beyond Milan but within either the UCSF or the up-to-7 criteria, this might have been affected by the type II error or, less probably, by the selection bias.

Performed analyses revealed that last pretransplant AFP was an independent risk factor for tumor recurrence; however, the effects varied with respect to fulfillment of different selection criteria. Notably, while the negative impact of AFP $>100 \mathrm{ng} / \mathrm{ml}$ was statistically and clinically significant in patients within the Milan criteria and beyond the Milan but within the extended 
criteria, no effects were observed in patients beyond the extended criteria. These results clearly support addition of biological criterion of AFP into the morphological criteria. Most importantly, the null rate of recurrence remained unchanged since the previous analysis, despite both doubling the number of patients and prolongation of the follow-up period for those included in the initial study. Moreover, the findings are further supported by incorporation of the Warsaw extended criteria in the decision-making process regarding selection of patients in the authors' department since 2013, potentially reducing the risk of selection bias.

Besides several selection criteria utilized in Asia, the concept of combining the biological tumor markers with morphological features is emerging in the Western perspective of liver transplantation for HCC. Important alternatives to the Warsaw extended criteria currently comprise the AFP model introduced by Duvoux et al. and the TTV/AFP introduced by Toso et al. ${ }^{31,32}$ Subsequent studies validated the AFP model with respect to both longterm outcomes and net health benefit and the TTV/AFP criteria with respect to long-term outcomes. ${ }^{43-46}$ In contrast to these highly relevant proposals of complete redefinition of the Milan criteria, the Warsaw proposal provides an option to expand them in a more conservative fashion, by using the well-established UCSF and up-to-7 criteria. Therefore, AFP cutoff of $100 \mathrm{ng} / \mathrm{ml}$ is used in fact as an exclusion criterion, to bring the risk of tumor recurrence associated with increasing tumor burden to or below the level provided by the Milan criteria. Presumably, the use of the Warsaw extended criteria would expand the pool of potential HCC recipients by approximately $20 \%$, similar to the TTV/AFP criteria and the UCSF criteria, yet lower to the up-to-7 criteria. $^{47}$

Recently, the pretransplant AFP slope has been reported to be a novel predictor of HCC recurrence after liver transplantation. ${ }^{48}$ Nevertheless, this was not confirmed by several other studies, including that of the authors of this manuscript. ${ }^{49,50}$ However, the last pretransplant AFP also provides a dynamic assessment of patients eligibility for transplantation, as the AFP values are subject to spontaneous or neoadjuvant treatment-related changes in the pretransplant period.

In conclusion, the results of the present study provide further evidence for the potential lack of negative effects associated with the use of the Warsaw expansion of Milan criteria in selection of patients with HCC for liver transplantation.

FUNDING The study did not receive funding.

CONFLICT OF INTEREST There are no conflicts of interests to declare.
OPEN ACCESS This article is distributed under the terms of the Creative Commons Attribution 4.0 International License (http:// creativecommons.org/licenses/by/4.0/), which permits unrestricted use, distribution, and reproduction in any medium, provided you give appropriate credit to the original author(s) and the source, provide a link to the Creative Commons license, and indicate if changes were made.

\section{REFERENCES}

1. Bruix J, Han KH, Gores G, Llovet JM, Mazzaferro V. Liver cancer: approaching a personalized care. J Hepatol. 2015;62(1 Suppl):S144-56.

2. Kumar A, Acharya SK, Singh SP, et al. The Indian National Association for Study of the Liver (INASL) consensus on prevention, diagnosis and management of hepatocellular carcinoma in India: the Puri recommendations. J Clin Exp Hepatol. 2014;4(Suppl 3):S3-26.

3. Chapman WC, Klintmalm G, Hemming A, et al. Surgical treatment of hepatocellular carcinoma in North America: can hepatic resection still be justified? J Am Coll Surg. 2015;220:628-37.

4. Seshadri RM, Besur S, Niemeyer DJ, et al. Survival analysis of patients with stage I and II hepatocellular carcinoma after a liver transplantation or liver resection. HPB (Oxford). 2014;16:1102-9.

5. Squires MH 3rd, Hanish SI, Fisher SB, et al. Transplant versus resection for the management of hepatocellular carcinoma meeting Milan Criteria in the MELD exception era at a single institution in a UNOS region with short wait times. J Surg Oncol. 2014;109:533-41.

6. Zheng Z, Liang W, Milgrom DP, et al. Liver transplantation versus liver resection in the treatment of hepatocellular carcinoma: a meta-analysis of observational studies. Transplantation. 2014;97:227-34.

7. Landman MP, Feurer ID, Pinson CW, Moore DE. Which is more cost-effective under the MELD system: primary liver transplantation, or salvage transplantation after hepatic resection or after loco-regional therapy for hepatocellular carcinoma within Milan criteria? HPB (Oxford). 2011;13:783-91.

8. Lai Q, Avolio AW, Lerut J, et al. Recurrence of hepatocellular cancer after liver transplantation: the role of primary resection and salvage transplantation in east and west. $J$ Hepatol. 2012;57:974-9.

9. Mazzaferro V, Regalia E, Doci R, et al. Liver transplantation for the treatment of small hepatocellular carcinomas in patients with cirrhosis. N Engl J Med. 1996;334:693-9.

10. Clavien PA, Lesurtel M, Bossuyt PM, Gores GJ, Langer B, Perrier A; OLT for HCC Consensus Group. Recommendations for liver transplantation for hepatocellular carcinoma: an international consensus conference report. Lancet Oncol. 2012;13:e11-22.

11. Bonadio I, Colle I, Geerts A, et al. Liver transplantation for hepatocellular carcinoma comparing the Milan, UCSF, and Asian criteria: long-term follow-up of a Western single institutional experience. Clin Transplant. 2015;29:425-33.

12. Fan J, Yang GS, Fu ZR, et al. Liver transplantation outcomes in 1,078 hepatocellular carcinoma patients: a multi-center experience in Shanghai, China. $J$ Cancer Res Clin Oncol. 2009; 135:1403-12.

13. Shirabe K, Taketomi A, Morita K, et al. Comparative evaluation of expanded criteria for patients with hepatocellular carcinoma beyond the Milan criteria undergoing living-related donor liver transplantation. Clin Transplant. 2011;25:E491-8. 
14. Yao FY, Ferrell L, Bass NM, et al. Liver transplantation for hepatocellular carcinoma: expansion of the tumor size limits does not adversely impact survival. Hepatology. 2001;33:1394-403.

15. Mazzaferro V, Llovet JM, Miceli R, et al. Predicting survival after liver transplantation in patients with hepatocellular carcinoma beyond the Milan criteria: a retrospective, exploratory analysis. Lancet Oncol. 2009;10:35-43.

16. Singal AK, Guturu P, Hmoud B, Kuo YF, Salameh H, Wiesner RH. Evolving frequency and outcomes of liver transplantation based on etiology of liver disease. Transplantation. 2013;95:755-60.

17. Nagai S, Yoshida A, Facciuto M, Moonka D, Abouljoud MS, Schwartz ME, Florman SS. Ischemia time impacts recurrence of hepatocellular carcinoma after liver transplantation. Hepatology. 2015;61:895-904.

18. Piardi T, Gheza F, Ellero B, et al. Number and tumor size are not sufficient criteria to select patients for liver transplantation for hepatocellular carcinoma. Ann Surg Oncol. 2012;19:2020-6.

19. Cheah YL, Chow PKH. Liver transplantation for hepatocellular carcinoma: an appraisal of current controversies. Liver Cancer. 2012;1:183-9.

20. Prasad KR, Young RS, Burra P, Zheng SS, Mazzaferro V, Moon DB, Freeman RB. Summary of candidate selection and expanded criteria for liver transplantation for hepatocellular carcinoma: a review and consensus statement. Liver Transpl. 2011;17(Suppl 2):S81-9.

21. Durand F, Belghiti J. Liver transplantation for hepatocellular carcinoma: should we push the limits? Liver Transplant. 2003;9:697-9.

22. Tanaka T, Kurosaki M, Lilly LB, Izumi N, Sherman M. Identifying candidates with favorable prognosis following liver transplantation for hepatocellular carcinoma: data mining analysis. J Surg Oncol. 2015;112:72-9.

23. Chaiteerakij R, Zhang X, Addissie BD, et al. Combinations of biomarkers and Milan criteria for predicting hepatocellular carcinoma recurrence after liver transplantation. Liver Transplant. 2015;21:599-606.

24. Lai Q, Avolio AW, Graziadei I, et al. Alpha-fetoprotein and modified response evaluation criteria in solid tumors progression after locoregional therapy as predictors of hepatocellular cancer recurrence and death after transplantation. Liver Transplant. 2013;19:1108-18.

25. Hu Z, Zhou J, Wang H, et al. Survival in liver transplant recipients with hepatitis B- or hepatitis C-associated hepatocellular carcinoma: the Chinese experience from 1999 to 2010. PLoS One. 2013;8:e61620.

26. Yang SH, Suh KS, Lee HW, et al. A revised scoring system utilizing serum alphafetoprotein levels to expand candidates for living donor transplantation in hepatocellular carcinoma. Surgery. 2007;141:598-609.

27. Zheng SS, Xu X, Wu J, et al. Liver transplantation for hepatocellular carcinoma: Hangzhou experiences. Transplantation. 2008;85:1726-32.

28. Taketomi A, Sanefuji K, Soejima Y, et al. Impact of des-gammacarboxy prothrombin and tumor size on the recurrence of hepatocellular carcinoma after living donor liver transplantation. Transplantation. 2009;87:531-7.

29. Takada $Y$, Ito T, Ueda M, et al. Living donor liver transplantation for patients with HCC exceeding the Milan criteria: a proposal of expanded criteria. Dig Dis. 2007;25:299-302.

30. Shindoh J, Sugawara Y, Nagata R, et al. Evaluation methods for pretransplant oncologic markers and their prognostic impacts in patient undergoing living donor liver transplantation for hepatocellular carcinoma. Transpl Int. 2014;27:391-8.

31. Duvoux C, Roudot-Thoraval F, Decaens T, et al. Liver transplantation for hepatocellular carcinoma: a model including $\alpha$ - fetoprotein improves the performance of Milan criteria. Gastroenterology. 2012;143:986-94.

32. Toso C, Asthana S, Bigam DL, Shapiro AM, Kneteman NM. Reassessing selection criteria prior to liver transplantation for hepatocellular carcinoma utilizing the Scientific Registry of Transplant Recipients database. Hepatology. 2009;49:832-8.

33. Grąt M, Kornasiewicz O, Lewandowski Z, et al. Combination of morphologic criteria and $\alpha$-fetoprotein in selection of patients with hepatocellular carcinoma for liver transplantation minimizes the problem of posttransplant tumor recurrence. World J Surg. 2014;38:2698-707.

34. Krawczyk M, Grąt M, Barski K, et al. 1000 liver transplantations at the Department of General, Transplant and Liver Surgery, Medical University of Warsaw: analysis of indications and results. Pol Przegl Chir. 2012;84:304-12.

35. Krawczyk M, Grąt M, Grąt K, et al. Evolution of the results Of 1500 liver transplantations performed In The Department Of General, Transplant And Liver Surgery Medical University Of Warsaw. Pol Przegl Chir. 2015;87:221-30.

36. Yao FY, Xiao L, Bass NM, Kerlan R, Ascher NL, Roberts JP. Liver transplantation for hepatocellular carcinoma: validation of the UCSF-expanded criteria based on preoperative imaging. Am J Transplant. 2007;7:2587-96.

37. Fernández JA, Robles R, Marin C, et al. Can we expand the indications for liver transplantation among hepatocellular carcinoma patients with increased tumor size? Transplant Proc. 2003;35:1818-20.

38. Decaens T, Roudot-Thoraval F, Hadni-Bresson S, et al. Impact of UCSF criteria according to pre- and post-OLT tumor features: analysis of 479 patients listed for HCC with a short waiting time. Liver Transplant. 2006;12:1761-9.

39. de Ataide EC, Garcia M, Mattosinho TJ, Almeida JR, Escanhoela CA, Boin IF. Predicting survival after liver transplantation using up-to-seven criteria in patients with hepatocellular carcinoma. Transplant Proc. 2012;44:2438-40.

40. Harimoto N, Shirabe K, Nakagawara H, et al. Prognostic factors affecting survival at recurrence of hepatocellular carcinoma after living-donor liver transplantation: with special reference to neutrophil/lymphocyte ratio. Transplantation. 2013;96:1008-12.

41. Xu X, Lu D, Ling Q, et al. Liver transplantation for hepatocellular carcinoma beyond the Milan criteria. Gut. 2015. doi: 10. 1136/gutjnl-2014-308513.

42. Unek T, Karademir S, Arslan NC, et al. Comparison of Milan and UCSF criteria for liver transplantation to treat hepatocellular carcinoma. World J Gastroenterol. 2011;17:4206-12.

43. Vitale A, Farinati F, Burra P, et al. Utility-based criteria for selecting patients with hepatocellular carcinoma for liver transplantation: a multicenter cohort study using the alpha-fetoprotein model as a survival predictor. Liver Transplant 2015;21:1250-8.

44. Varona MA, Soriano A, Aguirre-Jaime A, et al. Risk factors of hepatocellular carcinoma recurrence after liver transplantation: accuracy of the alpha-fetoprotein model in a single-center experience. Transplant Proc. 2015;47:84-9.

45. Toso C, Meeberg G, Hernandez-Alejandro R, Dufour JF, Marotta P, Majno P, Kneteman NM. Total tumor volume and alpha-fetoprotein for selection of transplant candidates with hepatocellular carcinoma: a prospective validation. Hepatology. 2015;62:158-65.

46. Grat M, Kornasiewicz O, Hołówko W, Lewandowski Z, Zieniewicz K, Paczek L, Krawczyk M. Evaluation of total tumor volume and pretransplantation $\alpha$-fetoprotein level as selection criteria for liver transplantation in patients with hepatocellular cancer. Transplant Proc 2013;45:1899-903.

47. Toso C, Kneteman NM, James Shapiro AM, Bigam DL. The estimated number of patients with hepatocellular carcinoma 
selected for liver transplantation using expanded selection criteria. Transpl Int. 2009;22:869-75.

48. Vibert E, Azoulay D, Hoti E, et al. Progression of alphafetoprotein before liver transplantation for hepatocellular carcinoma in cirrhotic patients: a critical factor. Am J Transplant. 2010;10:129-37.

49. Merani S, Majno P, Kneteman NM, Berney T, Morel P, Mentha $\mathrm{G}$, Toso C. The impact of waiting list alpha-fetoprotein changes on the outcome of liver transplant for hepatocellular carcinoma. $J$ Hepatol. 2011;55:814-9.

50. Grąt M, Krasnodębski M, Patkowski W, et al. Relevance of pretransplant $\alpha$-fetoprotein dynamics in liver transplantation for hepatocellular cancer. Ann Transplant. 2016;21:115-24. 